Aim of the study: The Ras-related tumour suppressor gene aplasia Ras homolog member I (ARHI) is downregulated in many types of cancer, including ovarian cancer and hepatocellular carcinoma. In the present study, we explore the expression level and role of ARHI in colon cancer. Moreover, the mechanisms that down-regulate expression of $\mathrm{ARHI}$ in colon cancer will be further investigated.

Material and methods: ARHI expression levels were evaluated with immunohistochemistry, reverse transcriptase-PCR, and western blot. Loss of heterozygosity (LOH), single strand conformation polymorphism (SSCP), and methylation-specific PCR (MSP) were used to study the mechanisms of ARHI down-regulation.

Results: Low expression of ARHI was observed in $61.7 \%$ (37/60) of colon cancer specimens. Compared with the paired noncancerous tissues, ARHI expression was significantly decreased in colon cancer tissues. Furthermore, low ARHI expression was significantly associated with worse differentiation degree and Dukes' stage $(P<0.05)$. Methylation-specific PCR assay revealed that the methylation rates of ARHI were $53.3 \%(16 / 30)$ and $46.7 \%$ (14/30) in ARHI CpG I and CpG II, respectively. Therefore, methylation of promoter may be involving in down regulation of $\mathrm{ARHI}$ expression.

Conclusions: These data highlight an important role for ARHI in colon cancer, which could be a therapeutic strategy against this malignancy.

Key words: colon cancer, tumour suppressor gene, $\mathrm{ARHI}$, methylation.

Contemp Oncol (Pozn) 2014; 18 (5): 329-333 DOI: $10.5114 /$ wo.2014.45764

\section{Loss of ARHI expression in colon cancer and its clinical significance}

\author{
Wei Wang ${ }^{1 *}$, Lili Chen ${ }^{2^{\star}}$, Qun Tang ${ }^{3}$, Yonggang Fan ${ }^{1}$, Xiaoru Zhang ${ }^{1}$, \\ Jingming Zhai $^{1}$
}

${ }^{1}$ Departments of General Surgery, the First Affiliated Hospital, Henan University of Science and Technology, Luoyang, Henan 471000, China

2Department of Pathology, the First Affiliated Hospital of Sun Yat-sen University, Guangzhou, Guangdong 510080, China

${ }^{3}$ Department of Pathology, Hunan University of Chinese Medicine, Changsha, Hunan 410208, China

"These authors contributed equally to this work.

\section{Introduction}

Colon cancer is one of the most significant malignancies in the gastrointestinal tract [1, 2]. Each year about 1,000,000 new cases of colon cancer are diagnosed [3]. Despite great breakthroughs in the treatment of colon cancer, recurrence and metastasis of tumour still affects the prognosis of patients. To increase survival rates of colon cancer patients, there is an urgent need for development of new therapeutic targets.

Aplysia Ras Homolog I (ARHI) is a small GTP-binding protein-encoding gene located on human chromosome 1 p31 [4, 5]. Interestingly, despite sharing 54-59\% homology with Ras, a proto-oncogene, ARHI is a tumour suppressor gene in a variety of tumours [6-8]. ARHI was the first tumour suppressor gene Ras superfamily. In cancer cells, expression of ARHI down-regulates cyclin D1 activity, induces p21WAF1/CIP1 expression, and inhibits cell growth in vitro and in vivo [9-11]. The effect of ARHI on cell growth depends on an NH2-terminal extension of the molecule that sets ARHI apart from Ras [12]. Moreover, ARHI can suppress the motility and invasiveness of tumour cells $[13,14]$.

An article reported that $\mathrm{ARHI}$ expression is weak or absent in breast cancer cells; however, ARHI is overexpressed in normal breast tissues [15]. A similar result was found in ovarian cancer [16]. Nevertheless, the effects of $\mathrm{ARHI}$ in colon cancer cells still remain unknown. Hence, in the present study, we aimed to explore the role of ARHI in colon cancer. Along with the development of research, we further investigate the molecular mechanism that down-regulates expression of ARHI in colon cancer. Gene inactivation is caused by DNA methylation, loss of heterozygosity ( $(\mathrm{LH})$, and mutation. Feng et al. reported that $\mathrm{LOH}$ and promoter methylation down-regulated ARHI expression in human ovarian cancers [17]. However, the inactivation cause of $\mathrm{ARHI}$ in colon cancer has not yet been reported. In the course of these studies, we found that the expression level of ARHI was highly correlated with promoter methylation.

\section{Material and methods}

Patients and tissue samples

Pathologic specimens with colon cancer were collected from the Department of Pathology of the First Affiliated Hospital, Henan University of Science and Technology. For immunohistochemistry analysis, these colon cancer cases included 60 paraffin-embedded colon cancer samples, with a mean age of 58 years (range, 42-71 years). Thirty fresh paired colon cancer and noncancerous colon tissues were used for methylation-specific PCR (MSP), LOH, and mutation detection. Informed consent on collection of samples was 
obtained by each patient. The study was approved by the Medical Ethics Committee of the first affiliated hospital of Henan University of Science and Technology.

\section{Immunohistochemistry}

4- $\mu \mathrm{m}$ sections were cut from the selected paraffin blocks. After washing the sections three times in phosphate-buffer saline (PBS), anti-ARHI (sc-30321, Santa Cruz, USA) was incubated overnight at $4^{\circ} \mathrm{C}$ and at $1: 200$ dilution. Labelling was detected by adding biotinylated secondary antibodies (Maxim-Bio, Fuzhou, China), avidin-biotin complex (Maxim-Bio), and diaminobenzidine (Maxim-Bio). The percentage positivity was scored as "0" (0-25\%), "1" (26-50\%), "2" (51-75\%), or "3" (> 75\%). The staining intensity was scored as "0" (no staining), "1" (weakly stained), "2" (moderately stained), or "3" (strongly stained). If the product of multiplication between staining intensity and the percentage of positive cells was $\leq 3$, it was considered as low expression of $\mathrm{ARHI}$, and high expression as a total score $\geq 4$.

\section{Reverse transcriptase-PCR}

Total RNA was prepared according to the manufacturer's recommendation, and 5ug RNA was used as a template for cDNA synthesis. Reverse transcription was performed for 15 minutes at $42^{\circ} \mathrm{C}$ in first strand buffer. The reaction was terminated by incubation for 5 minutes at $0^{\circ} \mathrm{C}$. The primers used in RT-PCR are listed in Table 1.

\section{Western blotting}

The samples were prepared by the sonication of scrapped subconfluent cells in PBS containing $1 \mathrm{mM}$ phenylmethylsulfonyl fluoride (PMSF) and $10 \mu \mathrm{g} / \mathrm{ml}$ aprotinin. Total protein of $30 \mu \mathrm{g}$ was boiled for 10 minutes before being loading onto $10 \%$ polyacrylamide gels and transferred to a polyvinylidene fluoride (PVDF) membrane. The membranes were incubated with ARHI antibody, followed by HRP-conjugated secondary antibody. Lastly, ARHI was visualised by enhanced chemiluminescence.

\section{Loss of heterozygosity and mutation analysis}

Allelic losses were analysed by PCR amplification with primers amplifying polymorphic microsatellites internal to $\mathrm{ARHI}$ at loci D1S2829. The primer sequences are shown in Table 1. PCR was performed on 80 ng of DNA for each sample. PCR products were denatured for 10 minutes at $100^{\circ} \mathrm{C}$ and then loaded on $8 \%$ denaturing gel. Cases were defined as $\mathrm{LOH}$ when an allele peak signal from tumour DNA was reduced by $50 \%$ compared with the normal counterpart.

Mutations of exon 1 and exon 2 were examined by PCR-SSCP. PCR amplification with primers is shown in Ta-

A

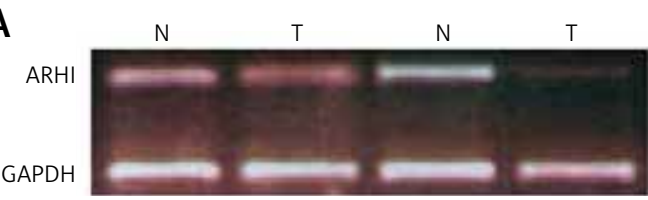

ble 1 . Then the PCR products were loaded on an $8 \%$ denaturing gel to examine mutation.

\section{Genomic DNA extraction and methylation-specific PCR}

To examine methylation of the promoter, we extracted DNA and treated genomic DNA with sodium bisulphite as reported previously [18]. In brief, $2 \mu \mathrm{g}$ of DNA was denatured by treatment with $2 \mathrm{M} \mathrm{NaOH}$ and modified with $3 \mathrm{M}$ sodium bisulphite for 16 hours. DNA samples were treated with CPGenomeTM DNA Modification Kit (CHEMICON, CAD) and resuspended in $25 \mu$ l water $2-\mu$ l aliquots, which were used as templates for PCR. For analysis of methylation of the ARHI promoter, MSP was performed with primers for the ARHI promoter, as shown in Table 1. PCR products were loaded onto $1 \%$ agarose gel and visualised under UV light.

\section{Statistical analysis}

The SPSS 11.5 software package for Macintosh (SPSS Inc, Chicago, IL) was used for all statistical analyses. Chisquared tests were used to evaluate the association between categorical variables and the ARHI expression. The Student $t$ test or the $t$ test for paired data was used to compare mRNA and protein expression levels in different groups. A significant difference was identified when the probability was less than 0.05 .

Table 1. Primers for RT-PCR, LOH, SSCP, and MSP

\begin{tabular}{|c|c|}
\hline Primers & Sequence (5'-3') \\
\hline \multicolumn{2}{|l|}{ RT-PCR } \\
\hline ARHI-F & TCTCTCCGAGCAGCGCA \\
\hline ARHI-R & CGTCGCCACTCTTGCTGTCG \\
\hline \multicolumn{2}{|l|}{$\mathrm{LOH}$} \\
\hline D1S2829-F & GTGGTTTATATGACTTACTGTGGG \\
\hline D1S2829-R & GCACNCCAGCCTAGGTA \\
\hline \multicolumn{2}{|l|}{ SSCP } \\
\hline Exon 1-F & ATTTGGAAAAGGGATTGG \\
\hline Exon 1-R & GTTGGGTTAGTTTTTTATAGTTGGTT \\
\hline Exon 2-F & GTAAGGGAGAAAGAAGTTAGA \\
\hline Exon 2-R & TACTATCСТAACAAAACССТC \\
\hline \multicolumn{2}{|l|}{ MSP } \\
\hline CpGl-F & GTTGGGTTAGTTTTTTATAGTTGGTT \\
\hline CpGI-R & AACCAAACAACCTAAAAAACAAATAC \\
\hline CpGII-F & GGGACACCGCTGATCGTTTA \\
\hline CpGII-R & ATAGGAAGATTAGAGATTAT \\
\hline
\end{tabular}

B

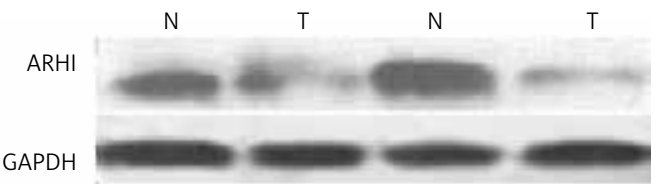

$N$-noncancerous tissues, $T$ - tumour

Fig. 1. Expression levels of ARHI in colon cancer tissues and the paired noncancerous tissues. A) ARHI mRNA expression; B) ARHI protein expression 


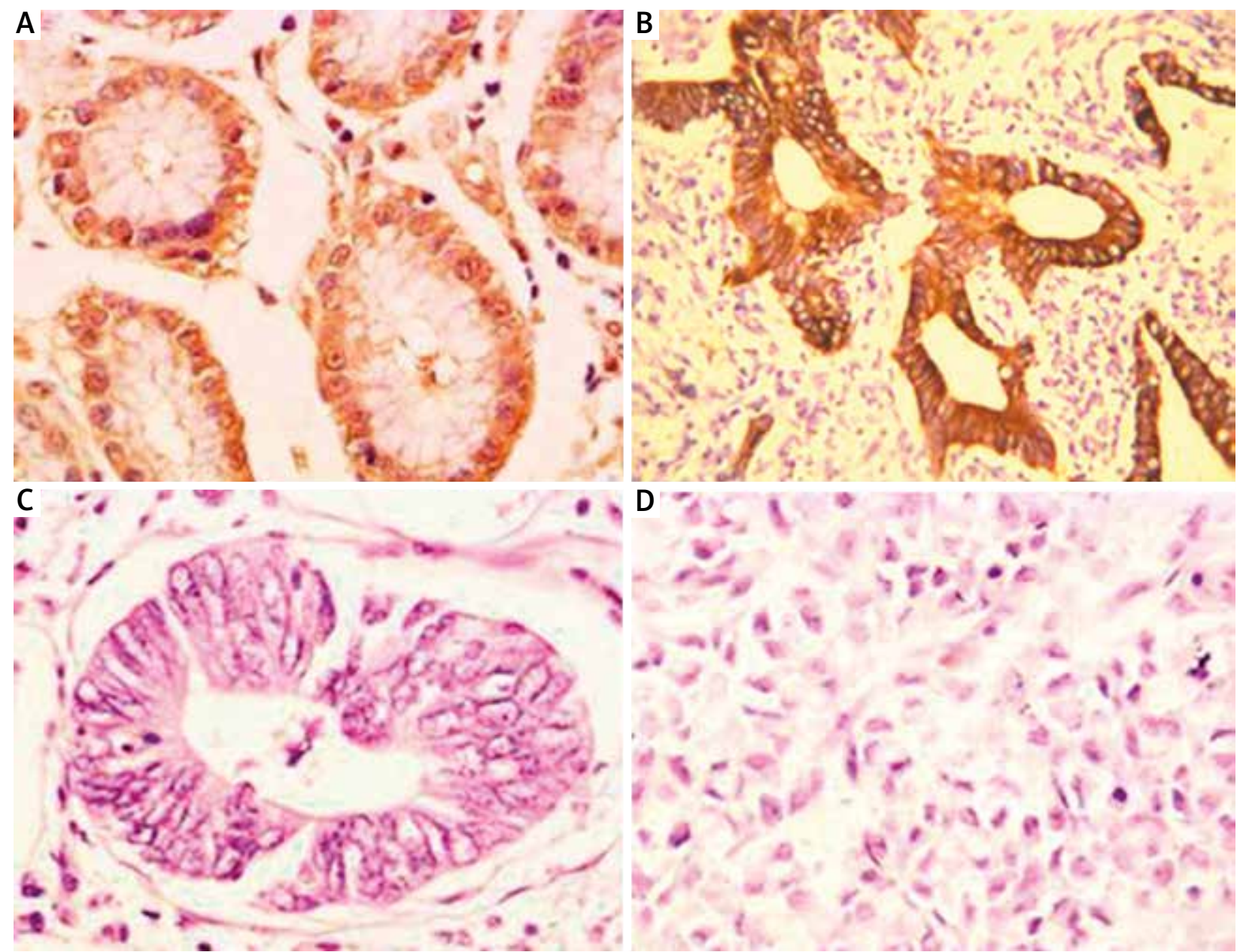

Fig. 2. Expression patterns of ARHI in colon cancer tissues. A) High expression of ARHI in normal colon tissues (200x); B) High expression of ARHI in colon cancer tissues (200x); C, D) Low expression of ARHI in colon cancer tissues (200x)

Table 2. Association between ARHI expression and clinicopathological features of the colon cancer patients

$\begin{array}{lcccc}\text { Variable } & \text { Cases } & \begin{array}{c}\text { ARHI expression } \\ \text { high }\end{array} & \boldsymbol{P} \\ & & \text { low } & \\ \text { Gender } & & & & \\ \quad \text { male } & 40 & 17 & 6 & 0.408 \\ \quad \text { female } & 20 & 23 & 14 & \\ \text { Age (years) } & & & & \\ \quad \leq 58 & 29 & 12 & 11 & 0.791 \\ \quad \text { > 58 } & 31 & 17 & 20 & \\ \text { Tumour size } & & & & \\ \quad \leq 2 \text { cm } & 24 & 12 & 11 & 0.177 \\ >\text { 2 cm } & 36 & 12 & 25 & \\ \text { Tumour differentiation } & & & & \\ \quad \text { Grade 1 } & 14 & 10 & 13 & 0.005 \\ \quad \text { Grade 2 + 3 } & 46 & 4 & 33 & \\ \text { Dukes' stage } & & & & \\ \text { A + B } & 21 & 13 & 10 & 0.011 \\ \text { C + D } & 39 & 8 & 29 & \\ \text { Lymph-node metastasis } & & & & \\ \quad \text { no } & 37 & 15 & 8 & 0.787 \\ \text { yes } & 23 & 22 & 15 & \end{array}$

\section{Results}

The expression levels of ARHI in colon cancer tissues

To investigate ARHI mRNA expression in colon cancer tissues, RT-PCR was used to analyse the expression of $\mathrm{ARHI}$ in six paired colon cancer and noncancerous colon tissues. The results suggested that ARHI was down regulated in all of the colon cancer tissues compared with the paired noncancerous tissues ( $P<0.05$, Fig. $1 A$ ). Moreover, we detected the expression of $\mathrm{ARHI}$ in six paired colon cancer and noncancerous colon tissues. Compared with paired noncancerous colon tissues, expression of ARHI protein was relatively weak in colon cancer tissues, and this result was consistent with the result of RT-PCR $(P<$ 0.05, Fig. 1B).

$\mathrm{ARHI}$ protein expression in colon cancer samples and normal colon tissues adjacent to cancer were detected by immunohistochemistry. We assessed the expression of ARHI in 60 paraffin-embedded colon cancer samples. The low immunoreactivity of ARHI was observed in $61.7 \%$ (37/60) of colon cancer samples, while $38.3 \%$ (23/60) of colon cancer specimens showed high expression of ARHI (Fig. 2). However, the ARHI-positive rate in adjacent noncancerous areas was 86.7\% (52/60). 


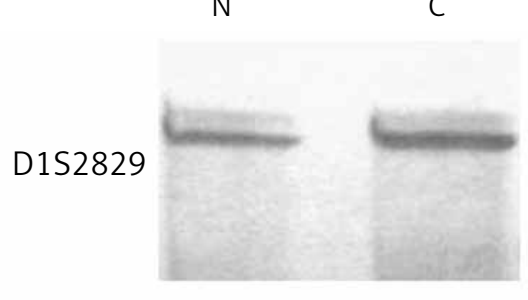

N

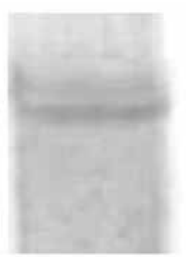

C

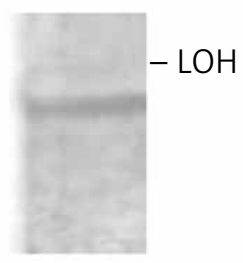

$\mathrm{N}$
C

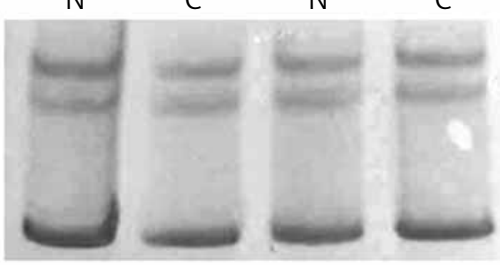

$N$-noncancerous tissues, $T$-tumour

Fig. 3. Results of LOH of ARHI (upper panel), 1 case of LOH was found (arrowhead); PCR-SSCP of exon 1 and exon 2 of ARHI (lower panel)

\section{Relationship between ARHI expression and clinicopathological factors}

To better elucidate the clinical significance of ARHI expression in colon cancer, we analysed the correlation between ARHI and clinicopathological factors of colon cancer. Table 2 shows that the down-regulation of ARHI was significantly associated with tumour differentiation ( $p=$ $=0.005)$ and Dukes' stage $(p=0.011)$. No significant association was found between ARHI expression and the other clinicopathological factors, such as gender, age, tumour size, and Lymph node metastasis.

\section{Loss of heterozygosity and mutation analysis}

To investigate the effects of $\mathrm{LOH}$ and mutation on down regulation of $\mathrm{ARHI}$ expression, a microsatellite marker, D1S2829, and PCR-SSCP were used in 30 cases. Representative results of $\mathrm{LOH}$ analysis were shown in Fig. 3. Loss of heterozygosity can be defined in a colon cancer sample in which one allele is decreased by at least $40 \%$ relative to the other. From all 30 cases, only 3 had $\mathrm{LOH}$ by using D1S2829. PCR-SSCP denaturing PAGE gel electrophoresis analysis of the ARHI gene was performed on colon cancer and adjacent noncancerous tissues. We screened the exon 1 and exon 2 of the $A R H I$ gene, and no mutation was found in colon cancer or adjacent normal colon lesions (Fig. 3).

\section{Frequent hypermethylation of $\mathrm{ARHI}$ in colon cancer}

To explore the mechanism of ARHI down-regulation in colon cancer, the methylation status of ARHI was measured by MSP in 30 colon cancer tissues. As shown in Fig. 4, the methylation rates of ARHI were $53.3 \%(16 / 30)$ and $46.7 \%$ (14/30) in ARHI CpG I and CpG II, respectively. Therefore, methylation of the promoter maybe involved in down regulation of $\mathrm{ARHI}$ expression.

\section{Discussion}

$\mathrm{ARHI}$ is a tumour suppressor gene that plays an important role in the proliferation and survival of cancer cells,
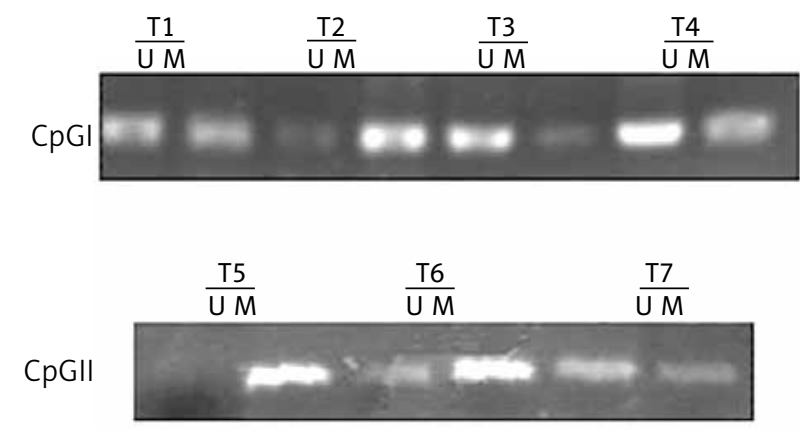

$U$ - unmethylation, $M$ - methylation

Fig. 4. Methylation analysis of ARHI CPG I and CPG II in colon cancer tissues

and which is frequently downregulated in various cancers [19-22]. The mechanisms of ARHI downregulation are very complex, including promoter methylation, $\mathrm{LOH}$, and histone acetylation. Despte extensive studies of ARHI in many other cancers, little is known about its role in colon cancer. Here we found that ARHI mRNA and protein expression levels were significantly reduced in colon cancer tissues compared with the paired noncancerous tissues, which is consistent with the Yuan et al. report on breast cancer [23]. Our results indicate that ARHI acts as a tumour suppressor gene in colon cancer. Immunohistochemistry assays suggest that low ARHI expression had significantly worse differentiation degree. Furthermore, low ARHI expression was significantly associated with Dukes' stage, which might demonstrate that the low expression of ARHI was correlated with more aggressive clinical stage in colon cancer.

Then we further investigated the mechanisms of ARHI downregulation. In the present study, $\mathrm{LOH}$ for $\mathrm{ARHI}$ was found only in 10\% (3/30) of all colon cancer cases, obviously lower than the previous reports in which $\mathrm{LOH}$ had been detected in $40 \%$ of ovarian cancers. This discrepancy may stem from the relatively small samples or different types of cancer. Therefore, LOH did not account for the major cause of the colon cancer in which ARHI was down regulated. 
Point mutation is a type of mutation that causes the replacement of a single base nucleotide with another nucleotide of the genetic material, and it could be an important mechanism for downregulation of gene expression. By using PCR-SSCP we did not find mutation of ARHI in exon 1 and exon 2. Thus, mutation also was not the main mechanism for ARHI downregulation.

Hypermethylation of the CpG islands of the promoter region is known to silence some genes as effectively as inactivation of the gene by mutations and deletions. Epigenetic changes of tumour-related genes through hypermethylation of $\mathrm{CpG}$ sites in the 5-promoter regions of various genes has been reported in different cancers. Thus, hypermethylation within a promoter of a tumour-suppressing gene may lead to inhibition of gene transcription and loss of its function. In the process of our study, methylation status of the ARHI promoter was examined in 30 colon cancer samples, and hypermethylation of ARHI CpG I and CpG II was 53.3\% (16/30) and 46.7\% (14/30), respectively, in colon cancer cases. The frequency of promoter hypermethylation of $\mathrm{ARHI}$ was relatively higher than that of ovarian cancers (31\%). Consequently, promoter methylation and inactivation of $\mathrm{ARHI}$ may play critical roles in the carcinogenesis of colon cancer.

To the best our knowledge, this is the first study evaluating the expression levels of ARHI mRNA and protein in colon cancer tissues and its correlation with clinicopathological factors. In addition, promoter hypermethylation is a major cause for downregulation of ARHI. ARHI may be a valuable biomarker for diagnosis and progression of colon cancer.

The authors declare no conflict of interest. This work was supported by the Initial Doctoral Funding of Henan University of Science and Technology.

\section{References}

1. Bartczak-Tomczyk M, Sałagacka A, Mirowski M, Jeleń A, Balcerczak E. Quantitative analysis of FJ 194940.1 gene expression in colon cancer and its association with clinicopathological parameters. Contemp Oncol 2013; 17: 45-50.

2. Waszkiewicz N, Zalewska-Szajda B, Chojnowska S, et al. Isoen zymes $\mathrm{A}$ and $\mathrm{B}$ of $\mathrm{N}$-acetyl- -D-hexosaminidase in tissue of colon cancer. Prz Gastroenterol 2012; 7: 374-8.

3. Weitz J, Koch M, Debus J, Höhler T, Galle PR, Büchler MW. Colorectal cancer. Lancet 2005; 365: 153-65.

4. YU Y, XU F, Peng H, et al. NOEY2 (ARHI), an imprinted putative tumor suppressor gene in ovarian and breast carcinomas. Proc Natl Acad Sci U S A 1999; 96: 214-9.

5. Luo RZ, Fang X, Marquez R, Liu SY, Mills GB, Liao WS, Yu Y, Bast $\mathrm{RC}$. ARHI is a Ras-related small G-protein with a novel N-terminal extension that inhibits growth of ovarian and breast cancers. On cogene 2003; 22: 2897-909.

6. Badgwell DB, Lu Z, Le K, et al. The tumor-suppressor gene ARH (DIRAS3) suppresses ovarian cancer cell migration through inhibition of the Stat3 and FAK/Rho signaling pathways. Oncogene 2012; 31: 68-79.

7. Zou CF, Jia L, Jin H, et al. Re-expression of ARHI (DIRAS3) induces autophagy in breast cancer cells and enhances the inhibitory effect of paclitaxel. BMC Cancer 2011; 11: 22.
8. Janssen EA, Øvestad IT, Skaland I, et al. LOH at 1p31 (ARHI) and proliferation in lymph node-negative breast cancer. Cell Oncol 2009; 31: 335-43.

9. Michieli P, Chedid M, Lin D, Pierce JH, Mercer WE, Givol D. Induction of WAF1/CIP1 by a p53- independent pathway. Cancer Res 1994; 54: 3391-5.

10. Schmider A, Gee C, Friedmann W, Lukas JJ, Press MF, Lichtenegger W, Reles A. p21 (WAF1/CIP1) protein expression is associated with prolonged survival but not with p53 expression in epithelial ovarian carcinoma. Gynecol Oncol 2000; 77: 237-42.

11. Anttila MA, Kosma VM, Hongxiu J, Puolakka J, Juhola M, Saarikoski S, Syrjänen K. p21/WAF1 expression as related to p53, cell proliferation and prognosis in epithelial ovarian cancer. Br J Cancer 1999; 79: 1870-8.

12. Luo RZ, Fang X, Marquez R, Liu SY, Mills GB, Liao WS, Yu Y, Bast RC. $A R H I$ is a Ras-related small G-protein with a novel N-terminal extension that inhibits growth of ovarian and breast cancers. Oncogene 2003; 22: 2897-909.

13. Zhao X, Li J, Zhuo J, Cai L. Reexpression of ARHI inhibits tumor growth and angiogenesis and impairs the mTOR/VEGF pathway in hepatocellular carcinoma. Biochem Biophys Res Commun 2010; 403: 417-21.

14. Wu X, Liang L, Dong L, Yu Z, Fu X. Effect of ARHI on lung cancer cell proliferation, apoptosisand invasion in vitro. Mol Biol Rep 2013; 40: 2671-8

15. Li Y, Liu M, Zhang Y, Han C, You J, Yang J, Cao C, Jiao S. Effects of $\mathrm{ARHI}$ on breast cancer cell biological behavior regulated by $\mathrm{mi}$ croRNA-221. Tumor Biol 2013; 34: 3545-54.

16. Lu Z, Luo RZ, Peng H, et al. Transcriptional and posttranscriptional down-regulation of the imprinted tumor suppressor gene ARHI (DRAS3) in ovarian cancer. Clin Cancer Res 2006; 12: 2404-13.

17. Feng W, Marquez RT, Lu Z, et al. Imprinted tumor suppressor genes $\mathrm{ARHI}$ and PEG3 are the most frequently down-regulated in human ovarian cancers by loss of heterozygosity and promoter methylation. Cancer 2008; 112: 1489-502.

18. Herman JG, Graff JR, Myöhänen S, Nelkin BD, Baylin SB. Methylation-specific PCR: A novel PCR assay for methylation status of CpG islands. Proc Natl Acad Sci U S A 1996; 93: 9821-6.

19. Méndez M, Custodio A, Provencio M. New molecular targeted therapies for advanced non-small-cell lung cancer. J Thorac Dis 2011; 3: 30-56.

20. Lu X, Qian J, Yu Y, Yang H, Li J. Expression of the tumor suppressor ARHI inhibits the growth of pancreatic cancer cells by inducing $G 1$ cell cycle arrest. Oncol Rep 2009; 22: 635-40.

21. Lin D, Cui F, Bu Q, Yan C. The expression and clinical significance of GTP-binding RAS-like 3 (ARHI) and microRNA 221 and 222 in prostate cancer. J Int Med Res 2011; 39: 1870-75.

22. Wang L, Hoque A, Luo RZ, et al. Loss of the expression of the tumor suppressor gene ARHI is associated with progression of breast cancer. Clin Cancer Res 2003; 9: 3660-6.

23. Yuan J, Luo RZ, Fujii S, et al. Aberrant methylation and silencing of $\mathrm{ARHI}$, an imprinted tumor suppressor gene in which the function is lost in breast cancers. Cancer Res 2003; 63: 4174-80.

\section{Address for correspondence}

\section{Jingming Zhai MD, PhD}

Departments of General Surgery

the First Affiliated Hospital Henan

University of Science and Technology

24 Jinghua Road, Luoyang, Henan 471000, P.R. China

e-mail: zhaijingming1@126.com

Submitted: 6.11 .2013

Accepted: 13.11.2013 